\title{
Charge Transport in the Dense Two-Dimensional Coulomb Gas
}

\author{
Dierk Bormannt \\ Institut für Physik der Universität Augsburg, Memminger Str. 6, D-86135 Augsburg, Germany \\ (Replaced 20 May 1997; final version to be published in Phys. Rev. Lett.)
}

\begin{abstract}
The dynamics of a globally neutral system of diffusing Coulomb charges in two dimensions, driven by an applied electric field, is studied in a wide temperature range around the Berezinskiu-KosterlitzThouless transition. I argue that the commonly accepted "free particle drift" mechanism of charge transport in this system is limited to relatively low particle densities. For higher densities, I propose a modified picture involving collective "partner transfer" between bound pairs. The new picture provides a natural explanation for recent experimental and numerical findings which deviate from standard theory. It also clarifies the origin of dynamical scaling in this context.
\end{abstract}

PACS numbers: 05.60.+w, 64.60.Ht, 74.25.Fy, 74.40.+k

The Coulomb gas in two dimensions (2D CG) is the generic model for topological excitations ("vortices") in $2 \mathrm{D}$ systems with a $\mathrm{U}(1)$ order parameter symmetry [i]. Some important examples are the $X Y$ model of $2 \mathrm{D}$ planar magnets [2], superfluid or superconducting films [1] 3], Josephson junction arrays [4] and, with some modifications, 2D melting [1, 3]. Vortices form a CG due to the characteristic, logarithmic form of their interaction potential. Furthermore, via a suitable choice of boundary conditions (an imposed current in the case of superfluid systems) a topological "electric field" $E$ can be applied on the vortices. For conceptual clarity, we shall use the $C G$ language throughout this article [5].

The globally neutral 2D CG in $E=0$ undergoes the famous Berezinskiu-Kosterlitz-Thouless (BKT) transition [2]. For temperatures $T<T_{\mathrm{BKT}}$, all particles are effectively bound in neutral pairs and the system is a dielectric insulator. At $T_{\mathrm{BKT}}$ the pairs start to thermally dissociate, leading to metallic screening and conduction. In 2D superfluid systems, this unbinding of vortices causes the transition between superfluid and normal phases.

Besides its equilibrium phase transition, also the $d y$ namical behavior of the 2D CG is of considerable interest, since, e.g., in any superfluid system, moving vortices are the main cause of dissipation. A few years after the BKT papers, a number of publications [6] 9] therefore extended the BKT pairing idea to nonequilibrium situations, studying in particular the current response to a static, homogeneous applied field $E$. Their common procedure was to depart from the BKT decomposition of the system into two components, "bound pairs" and "free" particles, and to add kinetic equations for the particle exchange between both; other interactions than the intra-pair one are cast into (both dielectric and metallic) screening of the latter. Ambegaokar et al. (AHNS) [9] extended the approach to an oscillating field $E$ and concluded that at high driving frequencies the response is dominated by the internal dynamics of individual bound pairs (as well as, above $T_{\mathrm{BKT}}$, thermally generated free particles) whereas at low enough or zero frequency it is essentially due to free particle drift only. From BKT theory it is known [1] that below $T_{\mathrm{BKT}}$ the charge correlation function decays algebraically as $r^{-\beta(T)}$ with distance $r$ where $\beta(T):=1 /(\tilde{\epsilon}(T) T), T$ being the CG temperature and $\tilde{\epsilon}(T)$ its static dielectric constant. The assumption of pure bound-pair reponse leads to algebraic decay in time as well with a dynamical exponent $z=2$ at all temperatures $T \leq T_{\text {Вкт }}[10]$. Field-induced pair dissociation allows for a nonvanishing static response below $T_{\mathrm{BKT}}$ : AHNS found a nonlinear current-voltage (IV) relation $j \propto E^{a}$ ( $j$ is the CG current density here [5]) with

$$
a(T)=\beta(T) / 2+1 .
$$

Right at the BKT transition, $\beta\left(T_{\mathrm{BKT}}^{-}\right)=4$ such that $a\left(T_{\mathrm{BKT}}^{-}\right)=3$, which together with $z=2$ satisfies the dynamical scaling [11] relation $a=z+1$.

Away from $T_{\mathrm{BKT}}$ however, doubt has recently been cast onto relation (11) by dynamical simulations of the classical $X Y$ model [12] as well as of the 2D CG in the continuous plane [13] and on a lattice [14], by a re-analysis of earlier experiments on layered superconductors [14, and also by new experiments on 2D proximity coupled Josephson junctions arrays [15]. A common conclusion of all of these investigations is that $a$ rather seems to obey the relation

$$
a(T)=\beta(T)-1,
$$

proposed by Minnhagen et al. (MWJO) [12]. In other work [16] better agreement with form (11) is found, so the issue is controversial. Right at $T=T_{\mathrm{BKT}}^{-}$, both expressions give a value of 3 , but (2) has a steeper increase with decreasing $T$. Assuming that the critical scaling relation $a=z+1$ holds in an extended region below $T_{\mathrm{BKT}}$, MWJO link (2) to a temperature dependent dynamical exponent

$$
z(T)=\beta(T)-2,
$$

providing further scaling arguments in favour of (3).

In this letter, I propose a concrete physical picture for the correlated particle motion in a regime of intermediate CG particle density $n$, which in a natural way leads to both relations (2), (3) independently. My picture is an extension of the "conventional" kinetic theory of Refs. [6] 9] and, accordingly, reproduces their results (11) and $z \equiv 2$ for the "true", asymptotic exponents in the limit 
of small $n$. Furthermore, the underlying collective processes require a sufficiently strong applied field $E$ (in the case of d.c. nonlinear response), or a sufficiently high frequency $\omega$ (in the case of a.c. linear response), respectively. Consequently, at fixed, finite $n$ we also recover (11) for small $E$, and $z=2$ for small $\omega$. The detailed crossover conditions are derived below; their direct observation in experiments or simulations is however lacking so far.

Following Refs. [6 9], we consider overdamped particle motion, described by a system of Langevin equations

$$
\frac{\mathrm{d} \boldsymbol{x}_{i}}{\mathrm{~d} t}=\mu_{0} q_{i}\left[\sum_{j(\neq i)} \boldsymbol{\nabla}_{i} V_{0}\left(\left|\boldsymbol{x}_{i}-\boldsymbol{x}_{j}\right|\right) q_{j}+\boldsymbol{\xi}_{i}+\boldsymbol{E}\right]
$$

where $\boldsymbol{x}_{i}$ are the CG particle positions and $q_{i} \in\{ \pm 1\}$ their charges $\left(\sum_{i} q_{i}=0\right) . \mu_{0}, V_{0}$ are the "bare" particle mobility and Coulomb potential; $V_{0}$ behaves as $V_{0}(r) \sim$ $\ln \left(r / r_{0}\right)$ for $r \gg r_{0}$, and $V_{0}(r) \sim 0$ for $r \ll r_{0}$ to prevent pair collapse [1]. The heat bath is represented by deltacorrelated gaussian random forces $\boldsymbol{\xi}_{i}$.

Let me briefly recall the conventional "free particle drift" picture [6 9] of $I V$ response to a static applied field $E$. The dynamically screened pair problem is described by a two-particle Langevin equation of the form (亩) with $\mu_{0}$ replaced by an effective charge mobility $\mu_{\text {eff }}$, and $V_{0}$ replaced by the statically screened pair potential $V_{\text {scr }}$ which we approximate as

$$
V_{\mathrm{scr}}(r) \approx\left\{\begin{array}{ll}
V_{0}(r) / \tilde{\epsilon} & , r<\ell_{\mathrm{scr}} \\
V_{0}\left(\ell_{\mathrm{scr}}\right) / \tilde{\epsilon}, & r>\ell_{\mathrm{scr}}
\end{array} .\right.
$$

$\ell_{\text {scr }}$ denotes the metallic screening length due to free charges and $\tilde{\epsilon}$ the macroscopic, static dielectric constant of bound pairs up to this scale $\ell_{\mathrm{scr}}$. Both $\mu_{\mathrm{eff}}$ and $\tilde{\epsilon}$ are actually weakly scale dependent quantities, but this does not change the conclusions in any essential way and we ignore it here. $\mu_{\text {eff }}$ only sets the overall timescale and thus does not enter the exponents $a$ or $z$.

Let us assume $T<T_{\mathrm{BKT}}$ (i.e., $\beta>4$ ) for the moment, such that for $E=0$ all particles are bound in pairs and there is no metallic screening. Under the action of $E \neq 0$, pairs dissociate and free particles recombine with finite rates. Standard transition state theory yields for the respective rates per area $\Gamma_{\text {diss }} \propto y E^{\beta}$ and $\Gamma_{\text {rec }} \propto n_{\mathrm{f}}^{2}$, where $y$ is the fugacity of pairs at a distance $r_{0}$ which controls the total particle density and $n_{\mathrm{f}}$ is the density of free particles. In the stationary state, both rates are equal. Assuming then that the current is caused by free particle drift in the field $E$, we arrive at

$$
j_{\mathrm{dr}} \propto n_{\mathrm{f}} E \propto y^{1 / 2} E^{\beta / 2+1},
$$

i.e., $a_{\mathrm{dr}}=\beta / 2+1$ [compare Eq. (11)] .

In order to see under which conditions the above argument holds, let us look at length scales in the problem which are relevant to transport. One is the separation $\ell_{E}=1 / \tilde{\epsilon} E$ at which a pair becomes unstable in the applied field $E$; pairs smaller than $\ell_{E}$ are (temporarily) bound. Another is the typical distance $\ell_{\mathrm{dr}}$ which a free particle drifts in the direction of $E$ until it recombines,

$$
\ell_{\mathrm{dr}}:=j_{\mathrm{dr}} / 2 \Gamma_{\mathrm{rec}}=j_{\mathrm{dr}} / 2 \Gamma_{\mathrm{diss}} \propto y^{-1 / 2} E^{-\beta / 2+1} .
$$

Since a neutral pair of size $<\ell_{E}$ is regarded as bound, a free particle density $n_{\mathrm{f}}>\ell_{E}^{-2}$ or, equivalently, a "drift length" $\ell_{\mathrm{dr}}<\ell_{E}$ clearly would be physically meaningless. Relations (6) and (7) imply $n_{\mathrm{f}} \ell_{E}^{2} \propto \ell_{E} / \ell_{\mathrm{dr}} \propto y^{1 / 2} E^{\beta / 2-2}$, so for sufficiently small $y$ or $E$ there is no contradiction. With increasing $y$ and $E$, however, $n_{\mathrm{f}} \ell_{E}^{2}$ and $\ell_{E} / \ell_{\mathrm{dr}}$ must exceed 1 at some point, implying a breakdown of the "free particle drift" picture and a crossover to a new "partner transfer" (PT) regime, where the constituents of a dissociated pair essentially have no opportunity anymore to drift freely, but immediately recombine with others. The $\mathrm{PT}$ regime may be visualized as a dense liquid of closeto-critical pairs of size $\approx \ell_{E}$ (by definition these cannot "overlap" each other), "transferring partners" among each other by thermally activated hopping [17]. The whole is of course immersed in a screening medium of smaller pairs, as discussed above.

In order to estimate the current in the $\mathrm{PT}$ regime, let us focus on the typical distance $\ell$ which a given particle separating from its partner is dragged along by $E$ until it binds to the next. This length shrinks from $\ell \sim \ell_{\mathrm{dr}} \gg \ell_{E}$ in the "drift" regime to its lower bound $\ell_{\mathrm{PT}} \sim x_{\mathrm{PT}} \ell_{E}$ (with a dimensionless constant $x_{\mathrm{PT}} \gtrsim 1$ ) in the PT regime. Assuming then that pair dissociation in the PT regime is still approximately described by transition state theory (implying $\Gamma_{\text {diss }} \propto y E^{\beta}$ ), we find

$$
j_{\mathrm{PT}}=2 \Gamma_{\mathrm{diss}} \ell_{\mathrm{PT}} \propto y E^{\beta-1},
$$

i.e., $a_{\mathrm{PT}}=\beta-1$ precisely as obtained in Refs. 12 14 [see Eq. (2)]! Note that an extension of the Kosterlitz renormalization procedure by including $E$ as a scaling variable 88.18] does not have a comparable effect, which indicates that the PT picture captures additional aspects of the strongly correlated dynamics at higher density.

MWJO 12 have attempted to reconcile the new relation $a_{\mathrm{PT}}=\beta-1$ with the "free particle drift" picture, postulating a modified dependence $\Gamma_{\text {rec }} \propto n_{\mathrm{f}}^{1+2 /(\beta-2)}$ of the recombination rate on the free particle density $n_{\mathrm{f}}$. If however, as we have argued here, the concept of free particles itself becomes questionable in the new regime, this type of explanation clearly cannot be adequate.

In the high temperature phase $(\beta<4)$ thermal pair dissociation leads to metallic screening, which provides a further characteristic length [1]

$$
\ell_{\mathrm{scr}} \propto n_{\mathrm{f}}^{-1 / 2} \propto y^{-1 /(4-\beta)} .
$$

As long as $\ell_{\mathrm{scr}}>\ell_{E}$, field induced pair dissociation dominates over thermal one and our arguments remain essentially valid, only the order of the "drift" and PT regimes is reversed: the PT regime now is at small $E$ (but still intermediate $y$ ). At still smaller $E$ such that $\ell_{\text {scr }}<\ell_{E}$ one eventually obtains an ohmic regime. 
The above results for $j(y, E)$ can be expressed by a scaling form of the nonlinear CG conductivity $\sigma_{\mathrm{CG}}$ [5],

$$
\sigma_{\mathrm{CG}}(y, E):=j(y, E) / E=\ell_{E}^{-2} \widetilde{\sigma}\left(\ell_{E} / \ell_{\mathrm{dr}}, \ell_{E} / \ell_{\mathrm{scr}}\right),
$$

where the scaling function $\widetilde{\sigma}$ depends on $\beta$ (but not $y$ ) and has the asymptotic forms $\widetilde{\sigma}(u, 0) \propto u$ for $u \ll 1$ ("drift") and $\propto u^{2}$ for $u \gg 1(\mathrm{PT})$, respectively, and $\widetilde{\sigma}(u, v) \propto v^{2}$ for any $u$ and $v \gg 1$ (ohmic regime).

Estimating quantitatively the prefactors in Eqs. (7) and (9), we find that the new PT regime extends to relatively small $y$ and $E$, so its predominance in experiments and computer simulations is not surprising. Using transition state theory to consistently estimate both pair dissociation [9] and recombination [6] rates we obtain

$$
\frac{\ell_{\mathrm{dr}}}{r_{0}} \approx \frac{\mathrm{e}^{-\beta / 2}}{\sqrt{8 \pi y}}\left(\frac{\ell_{E}}{r_{0}}\right)^{\beta / 2-1} .
$$

As to the screening length $\ell_{\text {scr }}$, a simple Debye-Hückel estimate [1] turns out to be quantitatively dissatisfactory since it implies $\ell_{\mathrm{scr}}<n_{\mathrm{f}}^{-1 / 2}$ at $\beta \approx 4$. Minimizing the grand canonical potential of a mixed system of bound pairs and free particles with respect to $\ell_{\text {scr }}$ yields instead [19]:

$$
\frac{\ell_{\mathrm{scr}}}{r_{0}} \approx\left(\frac{2 \pi}{\beta} \sqrt{y}\right)^{-2 /(4-\beta)} .
$$

Equations (11), (12) imply an extension of the PT regime to arbitrarily large $\ell_{E}$ (i.e., small $E$ ) in the vicinity of the BKT transition, since $\ell_{\text {scr }}$ diverges as $\beta \rightarrow 4^{-}$and, for $8 \pi y>\mathrm{e}^{-4}$ and any finite $\ell_{E}, \ell_{\mathrm{dr}} / \ell_{E}$ shrinks to below 1 as $\beta \rightarrow 4^{+}$. For typical 2D vortex systems 112,14, 15, $y$ and $\beta$ obey to a good approximation the "Villain" relation $\ln y \approx-\beta \pi / 2$ [1]. Figure 11 shows the resulting location of the different regimes in the $\beta-E^{-1}$ plane for some reasonable values of the (so far unknown) constant $x_{\mathrm{PT}}$ : in particular, in the vicinity of the BKT transition the asymptotic exponent $a_{\mathrm{dr}}=\beta / 2+1$ appears only at extremely small driving fields $E$ which probably are not reached in these investigations.

We now turn to dynamical correlations at $E=0$. Following Refs. [6 9], we assumed here that the motion of a given bound pair or free particle is essentially uncorrelated with the rest of the system, feeling it only through its screening properties. In particular, at $T<T_{\mathrm{BKT}}$, charge density correlations are governed by the internal dynamics of pairs [10]. The effective dynamical exponent $z$ for fluctuations with wavenumbers around $k$ can then be read off from the typical relaxation time $\tau(r) \propto r^{z}$ of pairs of size $r \propto k^{-1}$; it thus will depend on whether the relaxation process is dominated by "drift" or PT. The two mechanisms are illustrated in Fig. 2: (a) a member of a pair can simply drift towards the other under the action of its field, or (b) each of them can recombine with a member of another, smaller pair sitting in between them.
In case (a) each particle has to move a distance $\propto r$ pulled by the field $E_{\mathrm{P}} \propto 1 / r$ of its partner, which implies

$$
\tau_{\mathrm{dr}}(r) \propto r / E_{\mathrm{P}} \propto r^{2},
$$

i.e., $z_{\mathrm{dr}}=2$. This relaxation mechanism has been analyzed in detail by Ambegaokar and Teitel 10 and is certainly the dominant one in the limit of large $r$. In case (b) there is an area $\propto r^{2}$ within the big pair in which its dipole field has a strength $E_{\mathrm{D}} \propto 1 / r$ and thus induces the dissociation of smaller pairs with a rate per area $\Gamma_{\text {diss }} \propto y E_{\mathrm{D}}^{\beta}$, so the typical waiting time until such an event occurs behaves as [20]

$$
\tau_{\mathrm{PT}}(r) \propto 1 / r^{2} \Gamma_{\text {diss }} \propto y^{-1} r^{\beta-2},
$$

i.e., $z_{\mathrm{PT}}=\beta-2$ in agreement with (3). The four-body correlations implicit in this argument again underline the collective character of the PT mechanism. Comparing both relaxation times, $\tau_{\mathrm{dr}}(r) / \tau_{\mathrm{PT}}(r) \propto y r^{4-\beta}$, shows that below the BKT transition $(\beta>4)$ the "drift" mechanism is dominant at small $y$ or $k\left(\propto r^{-1}\right)$, whereas with increasing $y$ and $k$, PT takes over at some point.

(13), 14) imply dynamical scaling forms of the charge density correlation function which in turn, via the fluctuation-dissipation theorem, suggest a dynamical extension of (10) to $\sigma_{\mathrm{CG}}(y, E, \omega)$, including a further scaling variable $\ell_{\mathrm{dr}} / \ell_{\omega}\left(\ell_{\omega}:=\sqrt{\mu_{\mathrm{eff}} T / \omega}\right)$. For sufficiently large $y$ and $\omega$, the PT mechanism dominates, leading to a simplified form $\sigma_{\mathrm{CG}}(y, E, \omega) \sim \ell_{\omega}^{-2} \widetilde{\sigma}^{\prime}\left(\ell_{E}^{-z_{\mathrm{PT}}} / \ell_{\omega}^{2}\right)$ (the form of the scaling variable reflects the fact that $\tau_{\mathrm{PT}}\left(\ell_{E}\right) \propto \ell_{\mathrm{dr}}^{-2} \propto$ $\ell_{E}^{-z_{\mathrm{PT}}}$ in the $\mathrm{PT}$ regime). The validity of the "critical" scaling relation $a_{\mathrm{PT}}=z_{\mathrm{PT}}+1$ in a whole $T$ region below $T_{\mathrm{BKT}}$ (which was an assumption in the MWJO scaling argument!) now follows very naturally by the usual reasoning [11]. It expresses the fact that in the PT regime both dc charge transport and the decay of charge fluctuations are governed by the same mechanism, characterized by a single relevant length scale $\ell_{E} \propto 1 / E$.

In summary, I have described a new dynamical regime of the 2D CG in which charge transport predominantly occurs through direct particle transfer among bound pairs, instead of free-particle drift. It offers a natural interpretation of recent experimental and numerical findings on $2 \mathrm{D}$ vortex dynamics which deviate from "standard" AHNS theory [9]. In contrast to the more extreme view held by MWJO [12], I do, however, recover AHNS behavior asymptotically for low driving fields and frequencies; the new picture thus resolves the apparent contradiction between the MWJO and AHNS scenarios.

Further tests of this picture in experiments and numerical simulations are clearly needed; in particular, a direct observation of the predicted crossovers would be desirable. Another testable signature is the changed dependence of dc response (8) and relaxation times (14) on the pair fugacity $y$. I am not aware of any controlled way to tune $y$ independently of $\beta$ in real superconducting systems. In experiments on superfluid ${ }^{4} \mathrm{He}$ films, the vortex 
fugacity can be increased above the "Villain" value by adding ${ }^{3} \mathrm{He}$ impurities, which stick to the vortex cores and lower their energy 21. However, as we have seen, in order to observe the PT/ "drift" crossover a reduction of the fugacity would be needed instead.

In any event, in real systems a reliable determination of the effective CG temperature $T$ and thus of $\beta$ usually is an extremely difficult task. In contrast, CG simulations are natural test candidates since here $T, y$ are separately controllable model parameters (within certain limits this is also true for generalized $X Y$ type models [22]). Preliminary Monte Carlo data for a lattice CG [23] indeed support the proportionality (8) between $j$ and $y$ in the accessible parameter range.

The crossover at low $E$ [5] in the $I V$ characteristics should in principle be observable both in experiments and simulations. Figure 1 suggests to look for it at temperatures sufficiently far below $T_{\mathrm{BKT}}(\beta \gtrsim 5$, say), since the crossover value of $\ell_{E}$ diverges at $T_{\mathrm{BKT}}$. The same is true in principle for the low $\omega$ crossover in the dynamical correlations (i.e., in the ac linear response) although this one may be covered by other collective effects 22, 24].

I would like to thank P. Minnhagen, K. Holmlund, M. Nylen, O. Westman, H. Weber, H. Beck and S. R. Shenoy for clarifying conversations. This work was supported by grants from 'Svenska Forskningsrådet' and 'Deutsche Forschungsgemeinschaft'.

* Electronic address: bormann@physik.uni-augsburg.de

[1] P. Minnhagen: Rev. Mod. Phys. 59, 1001 (1987)

[2] V. L. Berezinskiǔ: Sov. Phys. JETP 34, 610 (1972); J. M. Kosterlitz, D. J. Thouless: J. Phys. C 6, 1181 (1973); J. M. Kosterlitz: J. Phys. C 7, 1046 (1974)

[3] D. R. Nelson, in Proceedings of the Les Houches Summer School, Session CXII (Elsevier, Amsterdam, 1996)

[4] Proceedings of the ICTP Workshop on Josephson Junction Arrays, Trieste, 1995, edited by H. A. Cerdeira and S. R. Shenoy [Physica (Amsterdam) 222B (1996)]

[5] For superconductors, the conventional quantities are as follows: (dc response) $I \propto E, V \propto j$; and (ac response) $\sigma(\omega) \propto\left[2 \pi \sigma_{\mathrm{CG}}(\omega)-\mathrm{i} \omega\right]^{-1}$.

[6] J. L. McCauley: J. Phys. C 10, 689 (1977)

[7] B. A. Huberman, R. J. Myerson, S. Doniach: Phys. Rev. Lett. 40, 780 (1978)

[8] R. J. Myerson: Phys. Rev. B 18, 3204 (1978)

[9] V. Ambegaokar, B. I. Halperin, D. R. Nelson, E. D. Siggia: Phys. Rev. Lett. 40, 783 (1978); Phys. Rev. B 21, 1806 (1980); B. I. Halperin, D. R. Nelson: J. Low Temp. Phys. 36, 599 (1979)

[10] V. Ambegaokar, S. Teitel: Phys. Rev. B 19, 1667 (1979)

[11] D. S. Fisher, M. P. A. Fisher, D. A. Huse: Phys. Rev. B 43, 130 (1991); A. Dorsey: Phys. Rev. B 43, 7575 (1991)

[12] P. Minnhagen, O. Westman, A. Jonsson, P. Olsson: Phys. Rev. Lett. 74, 3672 (1995).

[13] K. Holmlund, P. Minnhagen: Phys. Rev. B 54, 523 (1996)

[14] H. Weber, M. Wallin, H. J. Jensen: Phys. Rev. B 53, 8566 (1996)
[15] S. T. Herbert, Y. Yun, L. B. Gomez, S. Elhamri, K. Ravindran, D. B. Mast, R. S. Newrock: preprint (1996)

[16] M. V. Simkin, J. M. Kosterlitz: cond-mat/9610141, to appear in Phys. Rev. B

[17] A similar "transfer" between a bound pair and a free particle has implicitly been considered earlier [R. G. Petschek, A. Zippelius: Phys. Rev. B 23, 3483 (1981)] but in this situation only leads to a finite renormalization of the effective charge diffusion constant $\mu_{\text {eff }}$.

[18] S. Sujani, B. Chattopadhyay, S. R. Shenoy: Phys. Rev. B 50, 16668 (1994)

[19] Note that this form encloses the correct critical behavior $\ell_{\text {scr }} \propto \mathrm{e}^{\text {const } / \sqrt{T-T_{\mathrm{BKT}}}}$ because of the square root dependence $\tilde{\epsilon}(T)-\tilde{\epsilon}\left(T_{\mathrm{BKT}}\right) \propto \sqrt{T-T_{\mathrm{BKT}}}$ (see, e.g., [1]).

[20] Alternatively, this process can be described as a neutralizing PT current, flowing over a cross section $\propto r$ through the medium of smaller pairs, and driven by the electrical dipole field $E_{\mathrm{D}} \propto 1 / r$ of the big pair in that area. Equation (8) then yields $\tau_{\mathrm{PT}}(r) \propto 1 / r j_{\mathrm{PT}} \propto y^{-1} r^{\beta-2}$ again.

[21] A. N. Berker, D. R. Nelson: Phys. Rev. B 19, 2488 (1979); J. L. Cardy, D. J. Scalapino: Phys. Rev. B 19, 1428 (1979)

[22] A. Jonsson, P. Minnhagen: Phys. Rev. B 55, 9035 (1997)

[23] H. Weber: private communication

[24] M. Capezzali, H. Beck, S. R. Shenoy: Phys. Rev. Lett. 78, 523 (1997)

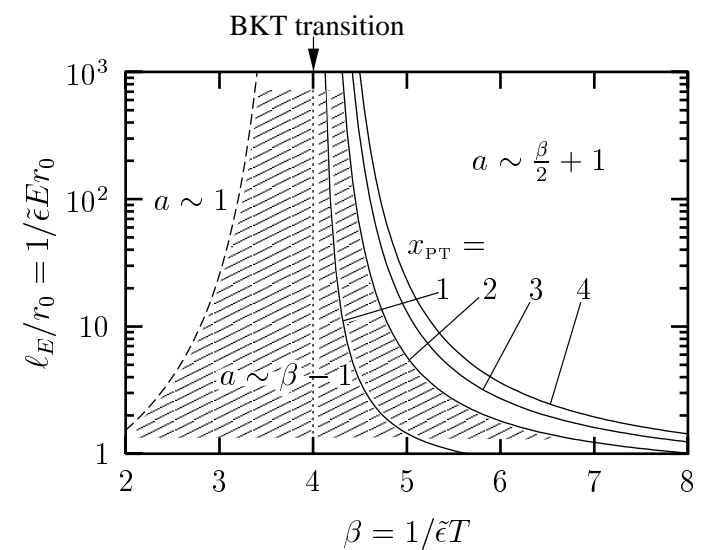

FIG. 1. Different $I V$ regimes in the $\beta-E^{-1}$ plane according to Eqs. (11), 12) and using the "Villain" relation $\ln y=-\beta \pi / 2$, for some reasonable values of $x_{\mathrm{PT}}$. The PT regime, characterized by the condition $\ell_{\mathrm{dr}} / x_{\mathrm{PT}}<\ell_{E}<\ell_{\mathrm{scr}}$, expands with increasing $x_{\mathrm{PT}}$ (shading is for $x_{\mathrm{PT}}=2$ ).
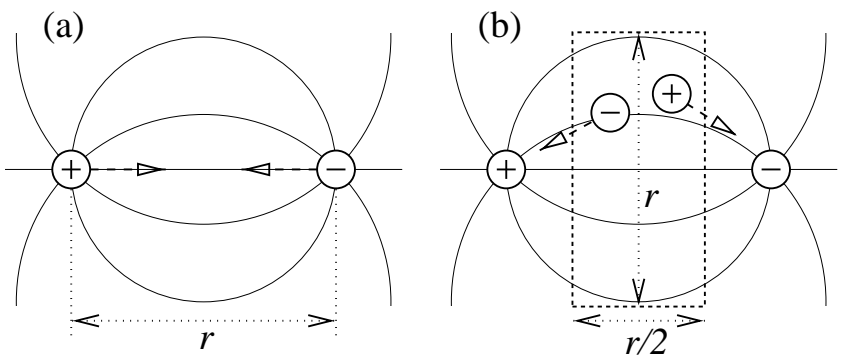

FIG. 2. Pair equilibration by (a) "drift" and (b) "partner transfer" (PT). In (b), the relevant region of small-pair dissociation, leading to $\mathrm{PT}$ relaxation of the big one, is framed by a dashed line. Electric field lines of the big pair are shown. 